\title{
LI-FI Become IOE: A Novel Architecture for LED
}

\author{
Umar Draz ${ }^{1}$, Tariq Ali, Sana Yasin, Ahmad Shaf, Ayesha Shakoor, and Umair Waqas \\ Computer Science Department, CIIT, Sahiwal, Pakistan
}

sheikhumar520@gmail.com

\section{Abstract}

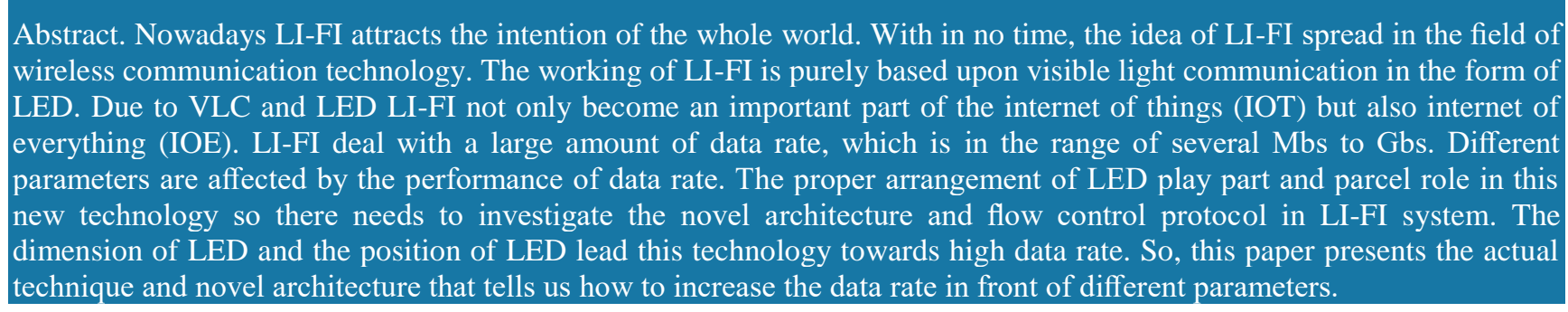

Keywords: VLC, IOE, IOT, data rate, OOS, dimensions of LEDs.

Received on 20 October 2017, accepted on 08 March 2018, published on 13 April 2018

Copyright (C) 2018 Umar Draz et al., licensed to EAI. This is an open access article distributed under the terms of the Creative Commons Attribution licence (http://creativecommons.org/licenses/by/3.0/), which permits unlimited use, distribution and reproduction in any medium so long as the original work is properly cited.

doi: 10.4108/eai.13-4-2018.154474

\section{Introduction}

The usage of LI-FI is increased in every walk of life. Due to LI-FI, the era of the internet of everything (IOE) has been started. So, there is need to flow control protocol for the usage of LI-FI. Currently, the congestion control is done through TCP using slow-start and AIMD (Additive Increase Multiplicative Decrease). Every TCP connection begins with congestion window that is already configured. The maximum segment size (MSS) is 4 [1]. By using slow-start procedure it investigates the network for currently available bandwidth. For every acknowledgment, the slow-start enhances the size of congestion window by one MSS. To do this, the result is doubling the window's worth of data is acknowledged. When a connection goes into slow-start process to observe the retransmission timeout and go out when the loss of the packet has been detected. When the TCP go out from this phase it still checks out the available bandwidth. When no packet loss than TCP increased the window size additively by $1 \mathrm{MSS}$. When congestion is detected decrease the half of window size. Various TCP flavors are available to check the reaction of congestion indication. For the homogeneous environment, these protocols are designed for the internet. But it is very interesting to know none of any protocol has been designed for the internet of things where things are connected using LI-FI which has high bandwidth and wasn't to access the internet which has comparatively very low bandwidth with the homogeneous environment $[2,3]$. There are some different flavors of TCP like TCP Tahoe, TCP Reno, TCP New Reno, TCP Sack (Selection Acknowledgement), XCP (Explicit Control Protocol), RCP (Rate Control Protocol), Three level ECN (Explicit Congestion Notification). All these flavors play our role to the flow control so that LED and its implementation architecture is flexible, dependable and moveable. LEDs data rate [4] depends upon several factors that directly or indirectly affect the performance of LI-FI $[1,5,6]$.

\section{LED Architectural Parameters}


In LI-FI technology the data rates (R) depend upon several parameters of LED. LED is the basic component of LI-FI, the data density and speed directly depending upon like total number of LED, size of LED, on-off switching of LED, distance of LED from receiver, height of LED.

\subsection{Data Rate Vs. Size of LED}

Size of LED directly affects the performance of data rates. With different sizes of LEDs, different data rates can be achieved. There are various size of LEDs were discussed in [7] is round about $1.8 \mathrm{~mm}, 3 \mathrm{~mm}$ or $5 \mathrm{~mm}$. All these are rounded top LED. Normally the rounded top LEDs are used to focus the light on a fixed point. In this way, the data is directly transmitted to a fixed point efficiently. With high intensity, the LED separate the light in a large area. For fast internet services and connectivity, micro chip LED can be used which generate data rates up to 150 Mbps. So, let me say that the size of LED is inversely proportional to the data rates. But the maximum data rate can be achieved by using $1 \mathrm{~nm}$ and $1 \mathrm{~m}$ LED that is considered to be a pixel in size [8] as shown in equation 1

$$
R \propto \frac{1}{S(L E D)}
$$

\subsection{Data Rate Vs. Number of LED}

It is a very linear relationship between these two parameters i.e; if the numbers of LED increased the data rates increased and vice versa. But one thing must be noted the number of LED is only increased in an available space of lamp. Proper adjustment of LED inside the lamp has promised to achieve maximum data rates. So, the direct relationship between data rate $(\mathrm{R})$ and the number of LEDs $(\mathrm{N})$ are shown in equation 2.

$$
R \propto N(L E D)
$$

\subsection{Data Rate Vs. OOS}

In LI-FI technology $[9,10]$ the 1 s and 0 s are produced. When the light is on it is 1 otherwise 0 . The function of On-Off switching of LEDs is at very high speed. The human eye can't detect the alterations. Different LEDs have different On-Off switching data rates, for example, the speed of organic LED is faster than micro LED and the micro LED is faster than normal LED. With the faster functionality of On-Off switching so in this way the possible order of transmitting the data is: Organic (LED) $>>$ Micro (LED) $>>$ Normal (LED). By using OFDM (Orthogonal Frequency Division Multiplexing), it permits the Micro-LED to maintain the millions of changes of light Intensity/Second. So the OOS is directly proportional to the data rates as shown in equation 3 .

$$
R \propto O(L E D)
$$

\subsection{Distance of LED from Receiver}

It is inversely proportional relation between distance and receiver. The reason is that light follows Line of Sight (LOS) direction and has monochromatic (same $\lambda$ ) nature. If the distance of the particular device which receives the LI-FI signals from LED, then the robustness of the signal is very weak. Normally ceiling of the room is about 10-15 feet and the light spread all around inside the room. But if the width of the room or any other building is increased as compared to its height than the light spread in all directions and the focus of light doesn't stable. So, the relationship between distance and the data rate is discussed in equation 4.

$$
R \propto \frac{1}{D(L E D)}
$$

\subsection{Height of LED from Receiver}

The height of LED with respect to installation and deployment, is a major factor that easily enhances or decrease the performance not only performance but also limited the data rate issues. The main reason is that the normal height of the room inside the building is several foots/meters. The installation of LED factor is very affective when the height of particular LED is maximum from the user. due to such deployment this is possible that total light does not be a focus for special purposes. The communication is inversely proportional to height of LEDs as shown in equation 5.

$$
R \propto \frac{1}{H(L E D)}
$$

\subsection{Distances between LED}

While deployment of LED in the ceiling of the room the distance factor is very critical because the maximum distance between two LEDs is not be increased as their light circles must be joined. There is the very strong reason behind this when the light spread along the floor the circle is formed as the particular user escape out the circle the light is dimmed so that the communication is lost. Therefore, for novel architecture, this idea is implemented to show that what the possible distance between two LEDs is.

2.7 R Vs. S(LED), N(LED), O(LED), D(LED) and $\mathrm{H}(\mathrm{LED})$ 
Now, we propose the relation of data rate $(\mathrm{R})$ with all possible parameters such as by combining all the equations we get equation 6 .

$$
R \propto \frac{N(L E D) O(L E D)}{S(L E D) D(L E D) H(L E D)}
$$

So, it can be concluded that the data rate can be calculated with the size and number of LEDs with distance, height and OOS function.

\subsection{Types of LEDs}

Different types of LEDs are available that are used for the visible light communication as described in [13]. Many LEDs have different characteristics with respect to quality and dimension.Fig. 1 illustrates the view of LEDs. We sum up the most used LEDs with VLC such as: MicroLED: It has high density parallel communication and potential to be used as display panels. Its speed is up to 1.5 Gbps [11]. Multichip LED: Combination of three o more LED chips have different colors such that RGB to generate white light. It has the capability to control the colors that depend upon the light intensity of different chips. Each color provides $15 \mathrm{MHz}$ bandwidth [12].

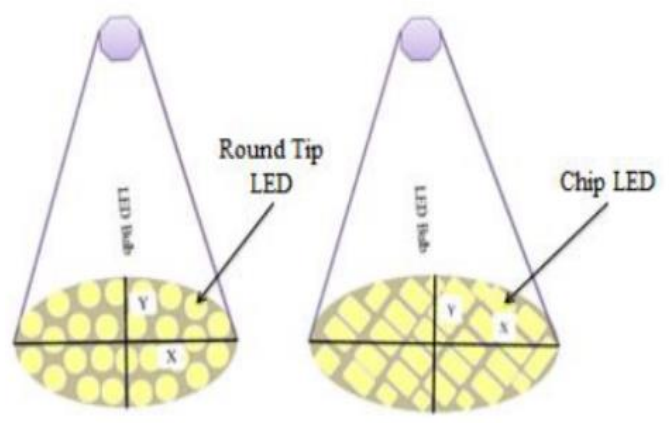

Fig. 1. Design of LEDs [3]

PC-LED: It is also called blue-LED with a phosphor layer coated on the top of it. Due to this factor, this LED is cheap but have the disadvantage of bandwidth limitation. The reason is that phosphor has a slow response. These are a further category like Pico chip, nano chip and simply chip LED. Resonant Cavity LED: To improve the highefficiency use RC-method. It's suited for optical communication applications. The domain of plastic optical fiber (POF) used this method for their bandwidth. It can be modulated in excess of $100 \mathrm{MHz}$.

\subsection{LED Circuit Diagram}

It is an electrical circuit diagram used to influence a LED. Design of the circuit in such a way that provides enough current to LED for the compulsory brightness but not at the maximum to prevent damaged the LED [14]. The drop of voltages will remain constant with extensive range of current. Fig. 2 represents the circuit diagram of LED.

$$
R=\frac{V s-V f}{I}
$$

$\mathrm{Vs}=$ Power supply voltage

$\mathrm{Vf}=\mathrm{LED}$ forward voltage

$\mathrm{I}=$ Desire current

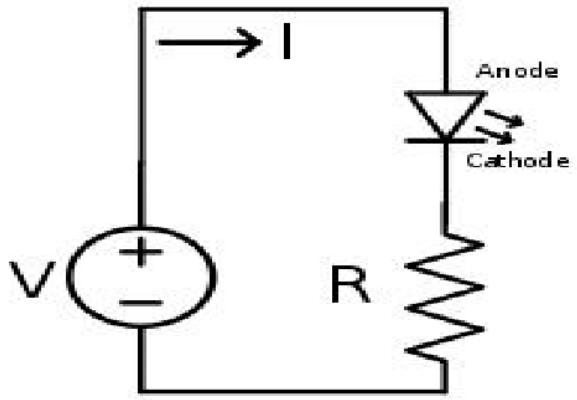

Fig. 2. LED circuit diagram

\subsection{Simulation Using DLT}

The results of this table show that resistance values depend on the forward voltage with the help of Derived Led Tool (DLT). When we increase the (vf) the resistance will be decreased and vice versa. So, it helps us to select the LED which has high resistance power. Table 1 shows the simulation result of LED tolerance power.

Table 1. Resistance of LED using DLT

\begin{tabular}{|l|l|l|l|l|}
\hline $\begin{array}{l}\text { Source voltages } \\
(\mathrm{vs})\end{array}$ & $\begin{array}{l}\text { Forward voltages } \\
\text { (Vf) }\end{array}$ & $\begin{array}{l}\text { Forward cur- } \\
\text { rent (If) }\end{array}$ & $\begin{array}{l}\text { Resistor } \\
\text { value }(\Omega)\end{array}$ & Power(W) \\
\hline 220 & 5 & 5 & $42 \mathrm{~K} \Omega$ & 1.075 \\
\hline 220 & 10 & 10 & $21 \mathrm{~K} \Omega$ & 2.5 \\
\hline 220 & 15 & 15 & $13.6 \mathrm{~K} \Omega$ & 3.075 \\
\hline 220 & 20 & 20 & $10 \mathrm{~K} \Omega$ & 4 \\
\hline 220 & 25 & 25 & $7.8 \mathrm{~K} \Omega$ & 4.875 \\
\hline 220 & 50 & 50 & $3.4 \mathrm{~K} \Omega$ & 8.5 \\
\hline 220 & 100 & 100 & $1.2 \mathrm{~K} \Omega$ & 12 \\
\hline 220 & 150 & 150 & $466 \Omega$ & 10.5 \\
\hline 220 & 200 & 200 & $100 \Omega$ & 4 \\
\hline 220 & 220 & 200 & 0 & 0 \\
\hline & & & & \\
\hline
\end{tabular}

\section{Use of concave and convex lens}

In front of LED, to enhance LED intensity lens play vital role either it is convex or concave. For spreading the light convex lens is helpful. All the rays of light that pass through the lens are spread out. Convexes, the rays, simply mean extending the focus of light. Fig. 3 represents the lens based LED. 


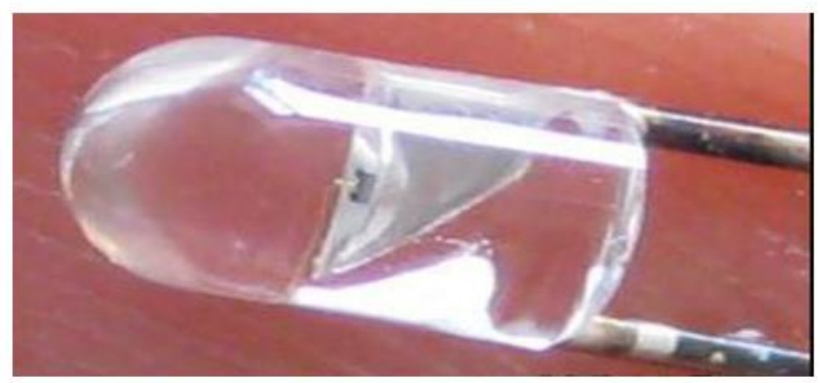

Fig. 3. Lenz based LED [16]

Actually, the major function of LED is tolerance power (TP) as discussed in [15]. Tolerance power (TP) is easily achieved with respect to digest maximum voltage. The maximum voltage is $5 \mathrm{~V}$. Fig. 4 is a view of the convex and concave lens that are joined in front of LED.

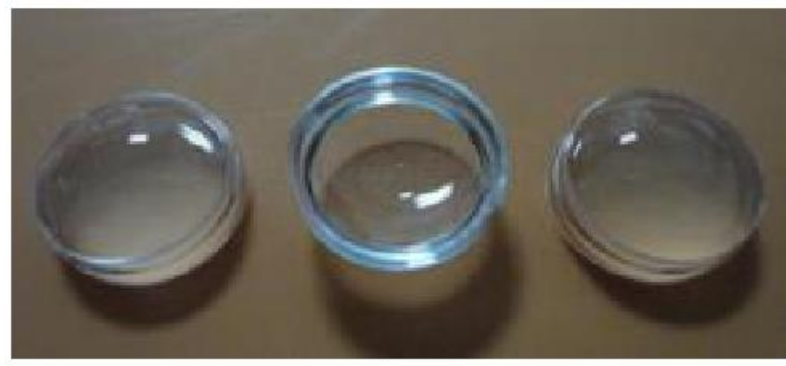

Fig. 4. Convex and concave lens [16]

\section{Novel Architecture for LEDs}

Basically, the main requirement for the novel architecture consists of the followings: MLU (Main LED Unit), AL (Agent LED), OOS (On-Off Switching) Dimension of bulb or LED lamp, Placement of positioning (PP) dimension and Internet structure design (ISD). The PP dimension further: Fixed position and moveable position. Fig. 5 shows that the fixed position of LEDs in round form and chip LED design. The fixed LED position determined the range of network communication at specific point where LED is attached.

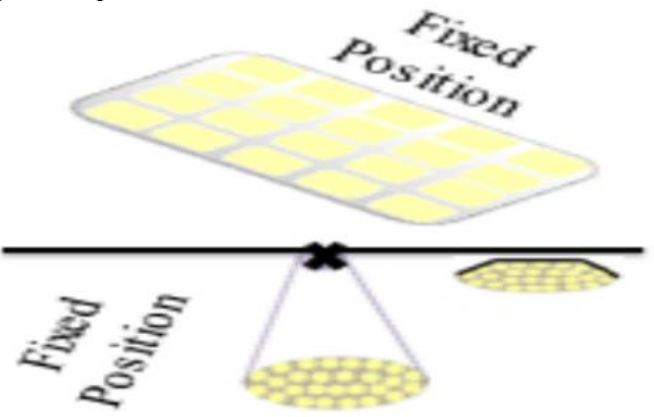

Fig. 5. Fixed position of LED

Fig. 6 shows the arrangements of LEDs that is movable according to need in particular place. The major advantage of moveable LED is that to select the required position according to requirements.

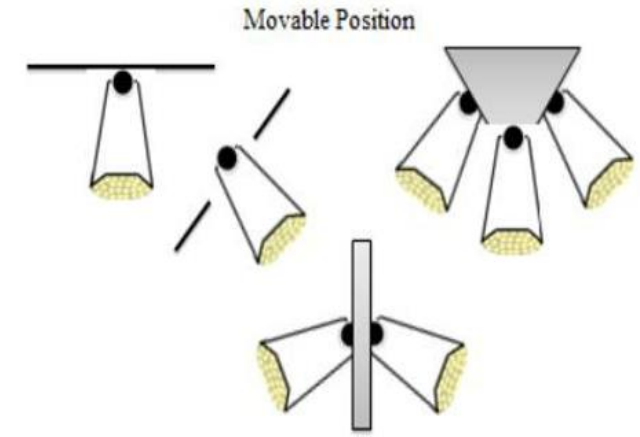

Fig. 6. Positions of LEDs

Rounded top led with a different size that affects the performance of communication, basically top is made for the proper focus of LED. Usually $1.8 \mathrm{~mm}$ LED is best suited when deploying within the series arrangement.

\subsection{Novel Architecture}

There is a novel architecture in which all LEDs are joined to ensure the parameters of size, height and the numbers of LEDs that describe the main LED units, further agent LEDs units to make a circle and radius of light for proper communication. Fig. 7 shows the novelty in the field of LI-FI with respect to LED architecture.

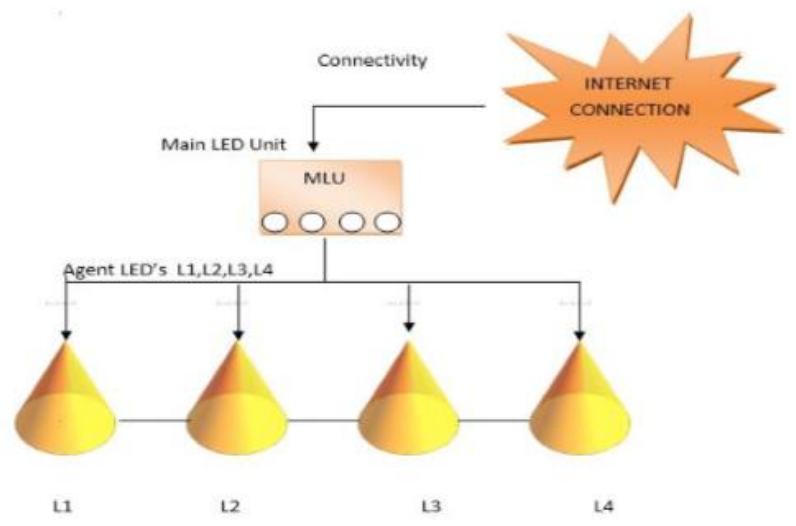

Fig. 7. Novel Architecture of LED

\section{Conclusion}

Now LI-FI is not in infant age. Towards green communication, the role of LI-FI becomes the open door of wireless communication. LED play part and parcel role in this innovation. But there is no fundamental architecture that is for deployment of LED. The proposed architecture of LED and realistic parameters plays vital role to improvement in this new innovation. The size, height, and OOS functions are those parameters that affected the performance of LI-FI communication. The 
data rate basically depends upon these various factors. The MLU and the agent LED are the main parts of the architecture. In this paper, the purposed future architecture of LI-FI and the arrangement of LEDs so that data rate could be maximum achieved. In future, we try to implement this novel architecture with these parameters and enhance the beauty of this architecture in welldisciplined manners.

\section{References}

1. R. R. Sharma, A. Sanganal, and S. Pati, "Implementation of a simple Li-Fi based system," International Journal of Computing and Technology, vol. 9, pp. 437-43, 2014.

2. Khandal, Dinesh, and Sakshi Jain. "Li-Fi (Light

Fidelity): The Future Technology in Wireless

Communication." International Journal of Information \& Computation Technology 4.16 (2014): 1687-1694.

3. R. Prakash and P. Agarwal, "The New Era of Transmission and Communication Technology: Li-Fi (Light Fidelity) LED \& TED Based Approach," International Journal of Advanced Research in Computer Engineering \& Technology (IJARCET), vol. 3, no. 2, 2014.

4. A. Shah, M. Shahzad, M. W. U. Siddiqi, and M. A. Khan, "Novel Architecture for Future Li-Fi Wireless Technology."

5. N. Navyatha, T. Prathyusha, V. Roja, and M. Mounika, "Li-Fi (Light Fidelity)-LED Based Alternative," International Journal of Scientific \& Engineering Research, vol. 4, no. 5, pp. 1039-1042, 2013. 6. J. Grubor, S. Randel, K.-D. Langer, and J. W. Walewski, "Broadband information broadcasting using LED-based interior lighting," Journal of Lightwave technology, vol. 26, no. 24, pp. 3883-3892, 2008. 7. K. P. Pujapanda, "LiFi integrated to power-lines for smart illumination cum communication," in Communication Systems and Network Technologies (CSNT), 2013 International Conference on, 2013, pp. 875-878: IEEE.

8. B. S. Rawat, B. Aggarwal, and D. Passi, "LI-FI: A new era of wireless communication data sharing," International Journal Of Scientific \& Technology Research, vol. 3, no. 10, 2014.

9. T. Marshall and M. Pashley, "LED collimation optics with improved performance and reduced size," ed: Google Patents, 2003.

10. R. R. Sharma and A. Sanganal, "Li-Fi Technology: Transmission of data through light," International Journal of Computer Technology and Applications, vol. 5, no. 1, p. $150,2014$.

11. N. Narendran and Y. Gu, "Life of LED-based white light sources," Journal of display technology, vol. 1, no. 1, pp. 167-171, 2005.

10 Umar Draz et al.
12. S. Pimputkar, J. S. Speck, S. P. DenBaars, and S. Nakamura, "Prospects for LED lighting," Nature Photonics, vol. 3, no. 4, pp. 180-182, 2009.

13. P. Bosua, "LED light bulb," ed: Google Patents, 2015. 14. X. Bao, G. Yu, J. Dai, and X. Zhu, "Li-Fi: Light fidelity-a survey," Wireless Networks, vol. 21, no. 6, pp. 1879-1889, 2015.

15. F. Aftab and S. Ali, "Light fidelity (LI-FI) based indoor communication system," arXiv preprint arXiv:1606.02831, 2016.

16. http://cn.made-in-china.com/tupian/yjmzjspMvEDuUCbIVW.html [Accessed on July 2017]: Time:01:14 AM. 